Ann. Biol. anim. Bioch. Biophys., I972, 12 (I), 125-138.

\title{
EFFETS D'UNE DÉFICIENGE ALIMENTAIRE EN LYSINE ET MÉTHIONINE SUR LES PERFORMANCES DE PONTE ET SUR LA TENEUR EN ACIDES AMINÉS LIBRES DU JAUNE D'CEUF
}

\author{
M. LARBIER, J.-C. BLUM et J. GUILLAUME \\ avec la collaboration technique de J.-P. Harscont \\ Station de Recherches avicoles, \\ Centre de Recherches de Tours, I. N.R. A., \\ 37 - Nouzilly
}

\section{RÉSUMÉ}

Après trois semaines de régime déficient soit en méthionine (lot $\mathrm{M}$ ), soit en lysine (lot L), soit en méthionine et lysine (lot ML), les performances chez la Poule pondeuse sont diminuées. Pour l'intensité de ponte, la diminution ( 5 à 7 p. Ioo) concerne seulement les poules carencées en méthionine. Le poids de l'œuf est réduit de 2,$9 ; 8,0$ et $5,3 \mathrm{~g}$ respectivement dans les lots $\mathrm{L}, \mathrm{M}$ et ML par rapport à celui des animaux témoins.

Proportionnellement, la déficience en méthionine réduit davantage le poids de l'albumen, la déficience en lysine le poids du vitellus. Dans le lot ML les performances de ponte, moins diminuées que dans le lot $M$, demeurent longtemps déprimées (plus de trois semaines) lorsque le régime complet est de nouveau distribué.

La consommation d'un régime déficient en acides aminés modifie les teneurs du vitellus en acides aminés libres. Ces variations quantitatives intéressent presque également tous les acides aminés libres. Après trois semaines de régime, on observe une diminution de 25 et $50 \mathrm{p}$. 100 respectivement dans les lots $\mathrm{M}$ et $\mathrm{L}$, tandis qu'au contraire, dans le lot $\mathrm{ML}$, la teneur totale augmente de $\mathrm{I}_{4}$ p. Ioo. Après distribution du régime complet pendant trois semaines, la concentration totale en acides aminés libres retrouve une valeur normale identique à celle des témoins, dans les lots $\mathrm{M}$ et $\mathrm{L}$ tandis que, dans le lot $\mathrm{ML}$, elle lui est inférieure de 25 p. roo.

L'équilibre des acides aminés de la ration pourrait influencer le renouvellement des protéines tissulaires et, partant, l'anabolisme de ponte ainsi que la teneur du vitellus en acides aminés libres.

\section{INTRODUCTION}

Mises en évidence par PUCHER en I927, les substances azotées non protéiques de l'œuf sont essentiellement localisées dans le jaune. Elles ont été identifiées comme étant des acides aminés libres par EMMANUEISSON (I95I) et par LEA et RHODES (I953). 
Leur dosage quantitatif proprement dit a été effectué grâce à l'utilisation des méthodes microbiologiques par WrLLIAMs et al. (I954) puis de la chromatographie sur colonne échangeuse d'ions par FiTzsimmons et WAIBEL (I967).

En nutrition aviaire, les acides aminés libres plasmatiques, depuis RICHARDSON, BLAYLOCK et LyMAN (I953), ou musculaires, depuis JonEs (I964) font l'objet de nombreuses recherches. Chez le Poulet, les teneurs en acides aminés libres du muscle reflètent, dans certaines conditions, la qualité des protéines de la ration (LARBIER, GUILIAUME et BLUM, I97I). Dans le cas du jaune de l'œuf, BRAY et KELLY (I966) ont découvert une relation entre la teneur du vitellus en lysine libre et le taux de cet acide aminé dans le régime. Cette relation serait linéaire pour des taux de lysine variant largement de part et d'autre du besoin.

Encouragés par ces résultats qui ne concernaient que la lysine, nous avons pensé que les concentrations des autres acides aminés indispensables présents sous forme libre dans le vitellus pouvaient également varier en fonction des apports alimentaires tant quantitatifs que qualitatifs d'acides aminés. Si cette hypothèse était vérifiée, nous disposerions là d'un test rapide pour apprécier l'efficacité des protides consommés par la Poule pondeuse.

Pour effectuer cette étude, nous avons fait varier les apports alimentaires de lysine et de méthionine et nous avons dosé chacun des acides aminés libres dans le jaune d'œuf.

\title{
MATÉRIEL ET MÉTHODES
}

\author{
I. - Animaux. Régimes
}

Les poules âgées de 30 semaines au début de l'essai sont issues d'un croisement commercial de type Rhode Island Red $\times$ Wyandotte ( $\mathrm{M}_{4} \mathrm{I}$, Selaf France). Élevées en cages individuelles, elles sont soumises à un éclairement de I 4 heures par nycthémère et bénéficient d'une alimentation ad libitum.

Pendant une période préexpérimentale de 4 semaines, tous les animaux reçoivent le même régime témoin $T$ à base de maïs et de tourteau d'arachide (tabl. I). Il est supplémenté en L-lysine $\mathrm{HCl}$ et $\mathrm{DL}$-méthionine de manière à couvrir les besoins protéiques de la pondeuse conformément aux normes du National Research Council (1966). Les teneurs en ces deux acides aminés ont été déterminées avant la supplémentation (tabl. I).

$\mathrm{Au}$ début de la période expérimentale, on constitue 4 lots de 8 animaux aussi homogènes que possible compte tenu du poids corporel, de l'intensité de ponte et du poids de l'œuf. La consommation alimentaire n'est pas retenue comme critère, car la valeur mesurée est faussée par le comportement des animaux (gaspillage). On distribue à trois lots des régimes expérimentaux, le quatrième (témoin) continuant à bénéficier d'une alimentation équilibrée (régime $T$ ). Tous ces régimes ne diffèrent que par leur supplémentation en L-lysine $\mathrm{HCl}$ et DL-méthionine (tabl. I). Le régime ML (régime de base) est déficient en L-lysine et DL-méthionine. Les régimes $M$ et $L$ sont obtenus en supplémentant ML respectivement en L-lysine et en DL-méthionine. Durant la période postexpérimentale, de même durée que les précédentes, tous les animaux reçoivent à nouveau le régime témoin.

Les différents lots sont désignés par la lettre servant à caractériser le régime de la période expérimentale.

$$
\text { 2. - Methodes analytiques }
$$

On pèse les animaux à chaque changement de régime et on enregistre la ponte ainsi que la consommation quotidienne individuelle tout au long de l'essai. Le poids de l'œuf et celui du vitellus sont déterminés directement; le poids de l'albumen est déduit par différence : œuf moins vitellus et coquille (lavée et séchée). 
Les teneurs en acides aminés libres du vitellus sont déterminées sur des œufs fraîchement pondus les $3^{\mathrm{e}}$ et $4^{\mathrm{e}}$ semaines de chacune des trois périodes préexpérimentale, expérimentale et postexpérimentale. Sur la ponte de chacune de ces semaines, on prélève au hasard deux œufs par poule. Les vitellus correspondant à l'ensemble des animaux d'un même lot sont réunis en deux échantillons pour l'analyse.

Les jaunes d'oufs, une fois débarrassés de leur membrane, sont d'abord dilués au I/2 dans du sérum physiologique. Les échantillons additionnés de norleucine sont extraits par l'acide picrique à I p. IOO (TALlan, MOORE et STEIN, I954) selon une technique d'épuisements successifs décrite par Pawlak et Pion (I968) à propos du muscle.

\section{TABLEAU I}

Composition des régimes utilisés

\begin{tabular}{|c|c|c|c|c|}
\hline Rćgime & $\mathrm{T}$ (témoin) & $\mathbf{L}$ & M & ML \\
\hline Mais $\ldots \ldots \ldots \ldots \ldots$. & 47,5 & 47,8 & 47,7 & 48,0 \\
\hline Tourteau d'arachide..... & 20,0 & 20,0 & 20,0 & 20,0 \\
\hline Amidon $\ldots \ldots \ldots \ldots \ldots$ & 17,0 & 17,0 & 17,0 & 17,0 \\
\hline Huile de maĩs & 3,0 & 3,0 & 3,0 & 3,0 \\
\hline Farine de luzerne $\ldots .$. & 2,0 & 2,0 & 2,0 & 2,0 \\
\hline $\begin{array}{l}\text { Mélange minéral et vitami } \\
\left.\text { nique }{ }^{1}\right) \ldots \ldots \ldots \ldots \ldots\end{array}$ & 10,0 & 10,0 & 10,0 & 10,0 \\
\hline L-lysine $\mathrm{HCl} \ldots \ldots \ldots$ & 0,3 & - & 0,3 & - \\
\hline DL-Méthionine ........ & 0,2 & 0,2 & - & - \\
\hline
\end{tabular}

Caractéristiques du régime $M L\left(^{2}\right)$

Énergie métabolisable. $3,06 \mathrm{kcal} / \mathrm{g}$

Matières azotées totales

15,8 p. 100

Lysine ........... 0,46 -

Méthionine ....... 0,22 -

Acides aminés soufrés. . $\quad 0,49$

(1) Apportant pour $100 \mathrm{~kg}$ d'aliment : carbonate de calcium $7 \mathrm{~kg}$, phosphate bicalcique $2 \mathrm{~kg}$, chlorure de sodium $500 \mathrm{~g}$, vitamine A $900000 \mathrm{UI}$, vitamine $\mathrm{D}_{3} 100000 \mathrm{UI}, \alpha$-tocophérol $2 \mathrm{~g}$, vitamine $\mathrm{B}_{2} 0,25 \mathrm{~g}$, BHT $3,5 \mathrm{~g}$, choline $100 \mathrm{~g}$, sulfate de manganèse $10 \mathrm{~g}$, iodure de potassium $0,05 \mathrm{~g}$, saccharose $365 \mathrm{~g}$.

(2) Déterminées par dosage sauf pour l'énergie métabolisable calculée à l'aide des tables.

L'acide picrique est éliminé par passage des extraits sur une colonne de résine échangeuse d'anions DowEx $2 \times 8$ (50 à Ioo mesh). L'effluent est concentré par évaporation sous vide puis ajusté à un volume connu à l'aide de tampon citrate $\mathrm{pH}: 2,2$.

Le dosage proprement dit est effectué en 22 heures au moyen d'un auto-analyseur TECHNICon selon la méthode décrite par PIEz et Morris (I960) et modifiée par HaMiLTon (I962).

\section{3. - Méthode statistique}

L'influence des changements de régime est analysée par comparaison des résultats obtenus pour chaque animal au cours des trois périodes, la préexpérience étant considérée comme période de référence. L'analyse statistique des résultats est effectuée sur la différence entre moyennes individuelles pendant les deux dernières semaines de chacune des périodes (préexpérience, expérience et postexpérience). Ces méthodes ont été appliquées par BLUM (I966) et LECLERCQ (I970) en d'autres occasions. 


\section{RÉSULTATS}

\section{I. - Résultats zootechniques (tabl. 2)}

Dans le lot témoin, l'intensité de ponte oscille entre 85 et $90 \mathrm{p}$. Ioo tandis que le poids corporel augmente régulièrement. Il en va de même du poids de l'œuf et de ses constituants. On vérifie donc que le régime témoin couvre les besoins de la poule et constitue, par conséquent, une référence valable. Il faut toutefois noter, que, contrairement à ce que l'on observe en général (RoMANoFf et RoManoff, I949), le poids du vitellus s'accroît proportionnellement davantage que celui de l'albumen : I5 et 7,5 p. Ioo respectivement.

Chez les animaux soumis au régime carencé en lysine (L), l'intensité de ponte et le gain de poids corporel sont de même ordre que dans le lot témoin. Toutefois, on observe deux effets en comparant les résultats des deux périodes, préexpérimentale et expérimentale. D'une part, l'accroissement du poids de 1'œuf est nettement plus faible : $2,6 \mathrm{p}$. Ioo contre $9,7 \mathrm{p}$. Ioo chez les témoins ; la différence est hautement significative $(\mathrm{P}<0,0 \mathrm{I})$. D'autre part, le développement relatif du vitellus $(+2,7$ p. Ioo) est devenu comparable à celui de l'albumen. Comparativement à ce que 1'on observe chez les témoins, la vitellogenèse est donc particulièrement réduite. Pendant la postexpérience on observe une compensation partielle des phénomènes précédents : le poids de l'œuf et surtout celui du vitellus augmentent plus rapidement que dans le lot témoin.

La déficience en méthionine, bien que ne modifiant pas la consommation, s'avère très néfaste. Elle entraîne un amaigrissement des pondeuses, une diminution significative du taux de ponte et surtout une chute du poids de l'œuf. Celle-ci atteint 4,5 p. Ioo par rapport aux résultats de la période préexpérimentale, alors que chez les animaux témoins, le poids de l'œuf a augmenté de $7,9 \mathrm{p}$. 100. La chute du poids de 1'œuf est due surtout à une diminution du poids de 1'albumen (6,8 p. roo par rapport à la préexpérience). La réduction quantitative qui intéresse le jaune est moins importante, quoique supérieure à celle observée lors de la carence en lysine. Tandis que les poids du vitellus et de l'albumen diminuent, celui de la coquille augmente ; c'est pourquoi la somme des variations du poids du vitellus et de l'albumen est supérieure à celle de l'œuf entier. Au cours de la postexpérience, les différences avec le lot témoin s'estompent : le poids de l'œuf augmente très rapidement, l'accroissement intéressant surtout 1'albumen (I3,7 p. Ioo). Il faut cependant noter que la réhabilitation n'est que partielle : le poids de l'œuf n'égale pas celui des témoins et l'intensité de ponte reste réduite à la fin de la postexpérience.

Pendant la phase expérimentale, les animaux du lot ML se comportent, sur nombre de points, de manière intermédiaire à ceux des lots $L$ et $M$. Le poids corporel et celui de l'ouf demeurent pratiquement invariables. Albumen et vitellus sont réduits quantitativement dans les mêmes et faibles proportions (I à 2 p. roo). On note cependant une diminution de l'intensité de ponte qui n'avait été observée que dans le lot M. Pendant la postexpérience, le lot ML se singularise par ses performances particulièrement faibles. Comme dans le lot $\mathrm{M}$, l'intensité de ponte reste déprimée. Le poids de l'œuf augmente nettement moins que dans les lots $\mathrm{M}$ et $\mathrm{L}$. 


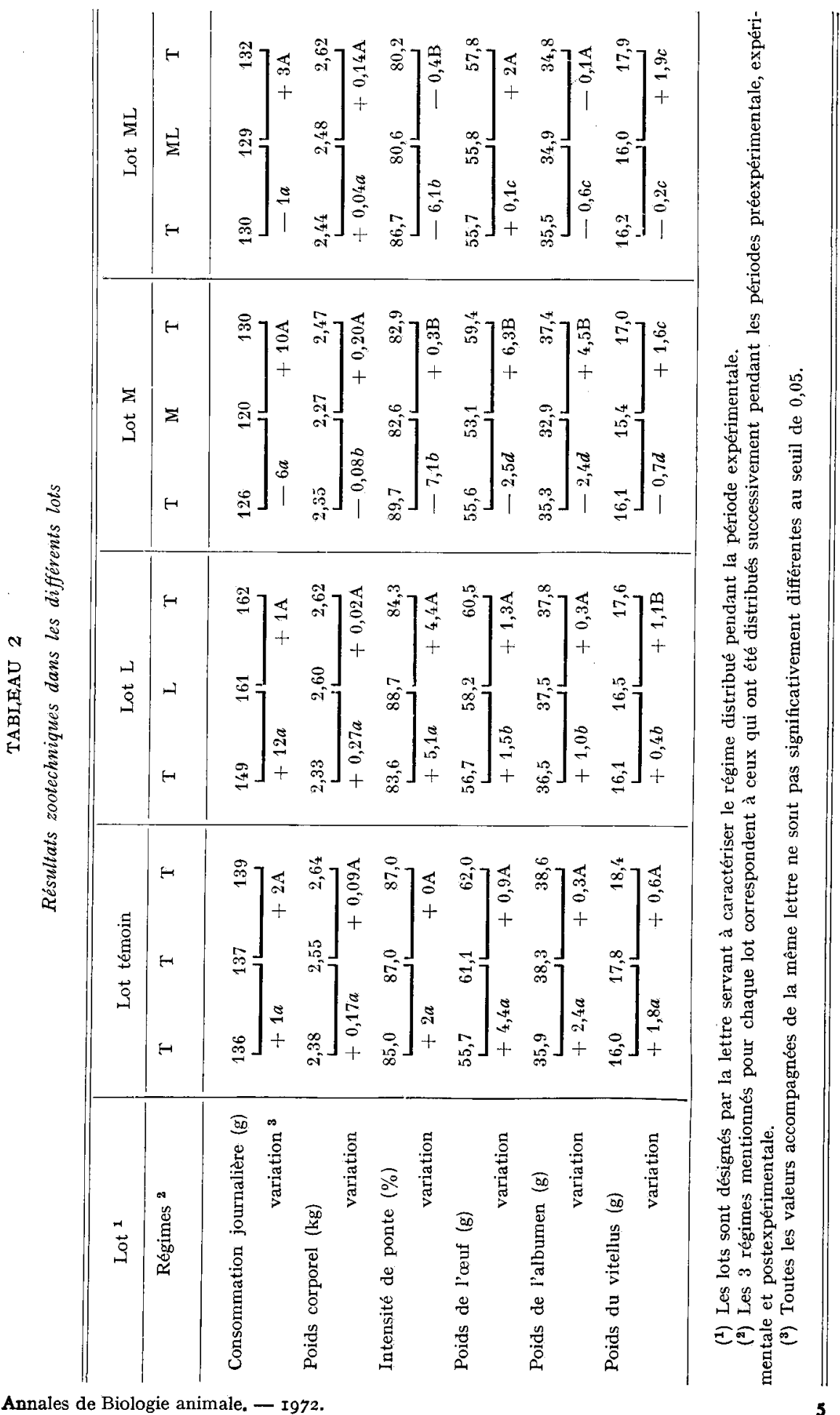


Cela est dû à l'absence de toute réhabilitation dans le cas de l'albumen, tandis que pour le vitellus la récupération est presque totale. Le meilleur profit retiré par l'animal au cours de la postexpérience concerne le poids corporel qui atteint celui des témoins.

\section{2. - Acides aminés libres du vitellus}

Nous avons identifié I 8 acides aminés libres mais nous n'avons pu en quantifier que I6 car les surfaces de pic correspondant à la cystine et à l'ornithine étaient trop faibles pour être intégrées. Dans nos conditions de dosage, nous n'avons pu détecter le tryptophane dont la présence sous forme libre dans le vitellus a été signalée par DUCAY, KLINE et MANDELEss (I960) qui utilisèrent la méthode de HorN et JoNEs (I945). Par ailleurs, nous constatons que le jaune d'œuf ne renferme pas de glutamine sous forme libre.

\section{a) Effets du régime témoin.}

La quantité totale d'acides aminés libres rapportée au volume de jaune d'œuf augmente légèrement au cours de l'essai chez les animaux nourris avec le régime témoin (tabl. 3). Quels que soient les lots d'animaux, les moyennes obtenues pendant

\section{TABLEAU 3}

Teneurs en acides aminés libres du vitellus (umole/ml) lorsque le régime est normal

\begin{tabular}{|c|c|c|c|c|c|c|}
\hline \multirow{4}{*}{$\begin{array}{l}\text { Acide aspartique... } \\
\text { Thréonine } \ldots \ldots \ldots\end{array}$} & \multicolumn{3}{|c|}{ Lot témoilı } & \multirow{2}{*}{\multicolumn{3}{|c|}{$\begin{array}{c}\text { Autres lots } \\
\text { pendant la préexpérience }\end{array}$}} \\
\hline & \multirow{2}{*}{$\begin{array}{c}\text { Préexp. } \\
2,11\end{array}$} & \multirow{2}{*}{$\begin{array}{c}\text { Expérience } \\
2,02\end{array}$} & \multirow{2}{*}{$\begin{array}{c}\text { Postexp. } \\
2,0 ;\end{array}$} & & & \\
\hline & & & & 2,07 & 2,10 & 2,09 \\
\hline & 1,99 & 2,19 & 2,29 & 2,10 & 2,12 & 2,00 \\
\hline Sérine $\ldots \ldots \ldots \ldots$ & 2,58 & 2,73 & 2,89 & 2,66 & 2,61 & 2,70 \\
\hline Acide glutamique... & 4,45 & 4,56 & 4,68 & 4,39 & 4,33 & 4,50 \\
\hline Proline........... & 1,29 & 1,44 & 1,60 & 1,30 & 1,25 & 1,28 \\
\hline Glycine ......... & 1,23 & 1,32 & 1,42 & 1,18 & 1,21 & 1,20 \\
\hline Alanine .......... & 1,33 & 1,53 & 1,74 & 1,29 & 1,30 & 1,33 \\
\hline Valine .......... & 1,68 & 1,74 & 1,80 & 1,70 & 1,64 & 1,72 \\
\hline Méthionine $\ldots \ldots \ldots$ & 0,59 & 0,55 & 0,60 & 0,57 & 0,61 & 0,53 \\
\hline Isoleucine $\ldots \ldots \ldots$ & 1,33 & 1,39 & 1,47 & 1,32 & 1,36 & 1,30 \\
\hline Leucine . . . . . . . . & 2,52 & 2,67 & 2,83 & 2,48 & 2,45 & 2,43 \\
\hline Tyrosine ........ & 1,54 & 1,60 & 1,66 & 1,52 & 1,54 & 1,49 \\
\hline Phénylalanine ... & 1,17 & 1,23 & 1,29 & 1,16 & 1,18 & 1,30 \\
\hline Lysine . . . . . . . & 2,20 & 2,40 & 2,60 & 2,17 & 2,15 & 2,20 \\
\hline Histidine $\ldots \ldots \ldots$ & 0,37 & 0,42 & 0,46 & 0,40 & 0,39 & 0,35 \\
\hline Arginine $\ldots \ldots \ldots$ & 1,45 & 1,57 & 1,69 & 1,42 & 1,42 & 1,50 \\
\hline Total ......... & 27,83 & 29,36 & 31,06 & 27,73 & 27,66 & 27,92 \\
\hline
\end{tabular}

la préexpérience sont extrêmement voisines. L'acide glutamique, la sérine, la leucine et la lysine sont les plus abondants tandis que la méthionine et l'histidine sont dans les plus faibles proportions. 
b) Effets d'une déficience en lysine: lot L.

Après deux semaines complètes de régime déficient en lysine ( $3^{\mathrm{e}}$ semaine), nous observons une légère diminution de la quantité totale d'acides aminés libres du vitellus (tabl. 4). Cet effet ne semble pas porter sur un acide aminé déterminé, la proportion de lysine en particulier reste sensiblement constante.

Après trois semaines de carence ( $4^{\mathrm{e}}$ semaine), la quantité totale d'acides aminés libres accuse une diminution rapide de 50 p. Ioo qui concerne inégalement les différents acides aminés. On observe une augmentation relative des acides glutamique et aspartique parallèlement à une diminution légère, il est vrai, des acides aminés basiques, de la méthionine et de la sérine.

Au cours de la postexpérience, la quantité totale d'acides aminés libres remonte progressivement pour atteindre à la fin de l'essai le niveau du lot témoin. L'ingestion de l'aliment complet annule donc, en quatre semaines, les effets de la déficience en lysine.

\section{c) Effets d'une déficience en méthionine: lot $M$.}

La déficience en méthionine affecte davantage la quantité totale d'acides aminés libres du vitellus que la teneur en un acide aminé particulier (tabl. 5). Nous retrouvons là, mais à un moindre degré, un effet dépressif comparable à celuti de la déficience en lysine. Cette fois encore, la diminution intéresse inégalement les différents acides aminés : légère augmentation de la proportion de sérine et appauvrissement en méthionine. Les teneurs relatives des acides glutamique et aspartique ne subissent aucune modification.

Au cours de la postexpérience, la quantité totale d'acides aminés libres du vitellus augmente sans toutefois atteindre le niveau observé chez les animaux témoins. Le régime déficient en méthionine a donc un effet plus durable que celui dans lequel la lysine constitue le facteur limitant. La différence peut être liée aussi bien au degré de 1a carence qu'à la nature de l'acide aminé concerné.

\section{d) Effets de la déficience simultanée en lysine et méthionine: lot ML.}

Nous observons (tabl. 6) une augmentation surprenante (ro p. roo) de la teneur de tous les acides aminés pendant la période expérimentale. Cet effet s'accentue pendant les deux premières semaines de la postexpérience. La $4^{e}$ semaine, une importante diminution ramène la quantité totale des acides aminés libres du vitellus à une valeur inférieure de $25 \mathrm{p}$. Ioo à celle enregistrée pendant la préexpérience chez les mêmes animaux.

\section{DISCUSSION}

Compte tenu des performances enregistrées, on dispose de quelques informations sur les besoins de la poule pondeuse M $4 \mathrm{r}$. Pour une consommation quotidienne de 380 à $480 \mathrm{kcal}$ d'énergie métabolisable, le besoin en lysine dépasse certainement les 0,50 p. Ioo préconisés par le National Research Council (Ig66) lorsque le régime apporte $3000 \mathrm{kcal} \mathrm{EM} / \mathrm{kg}$. Nos résultats témoignent d'une véritable carence pour un apport alimentaire égal à 0,46 p. Ioo (teneur déterminée par dosage). 
M. LARBIER, J.-C. BLUM, J. GUILLAUME

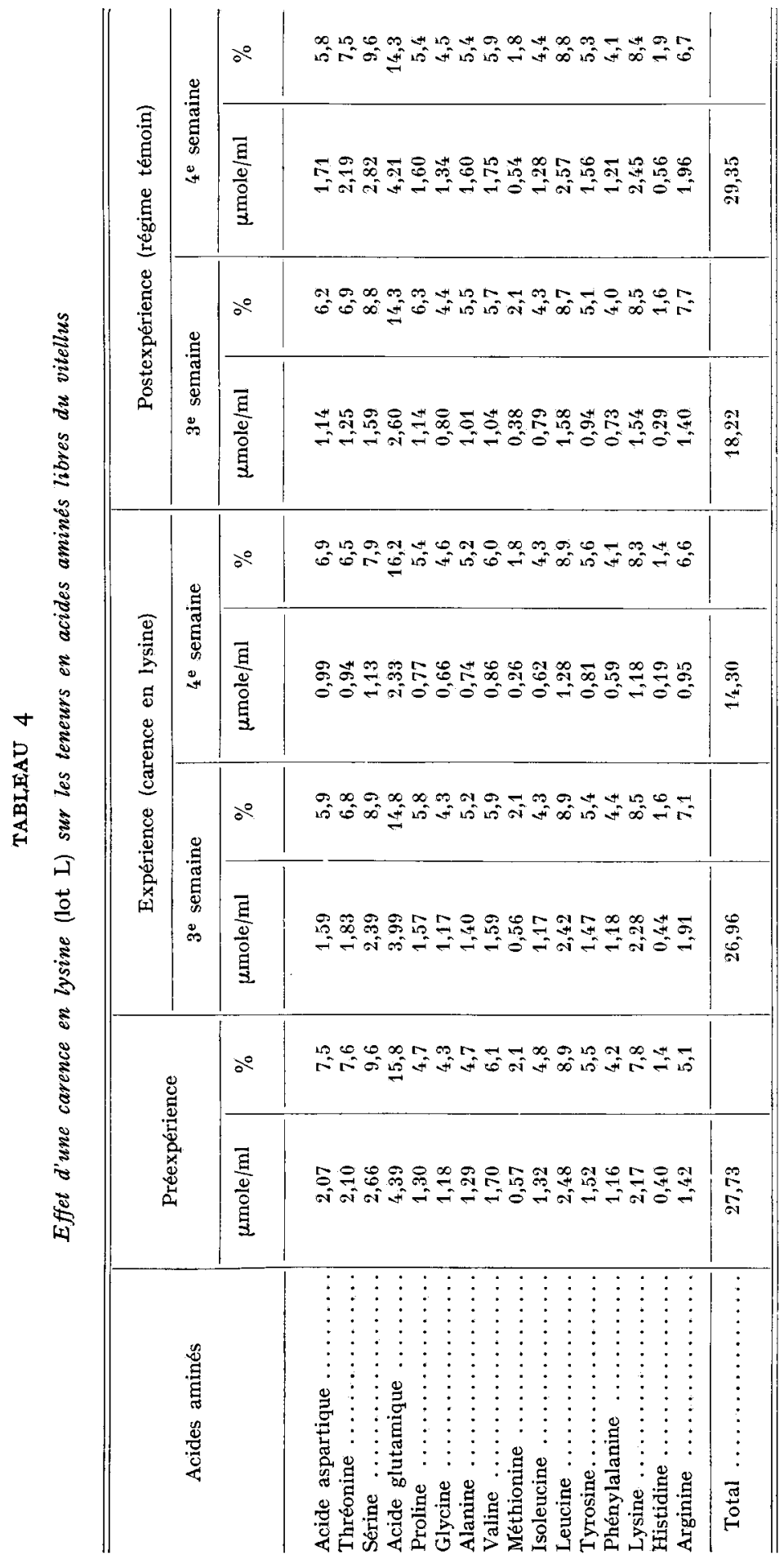




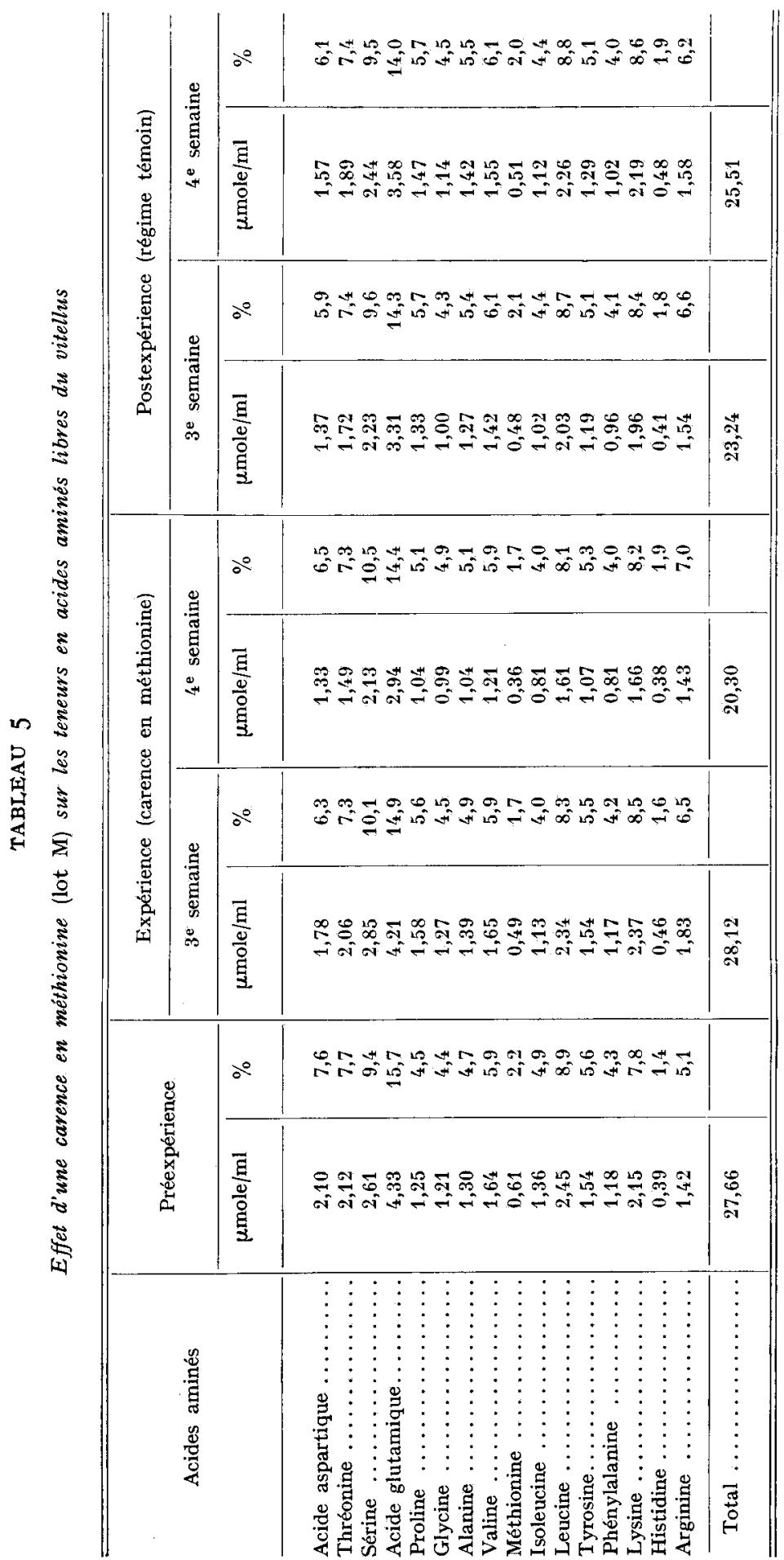


M. IARBIER, J.-C. BLUM, J. GUILLAUME

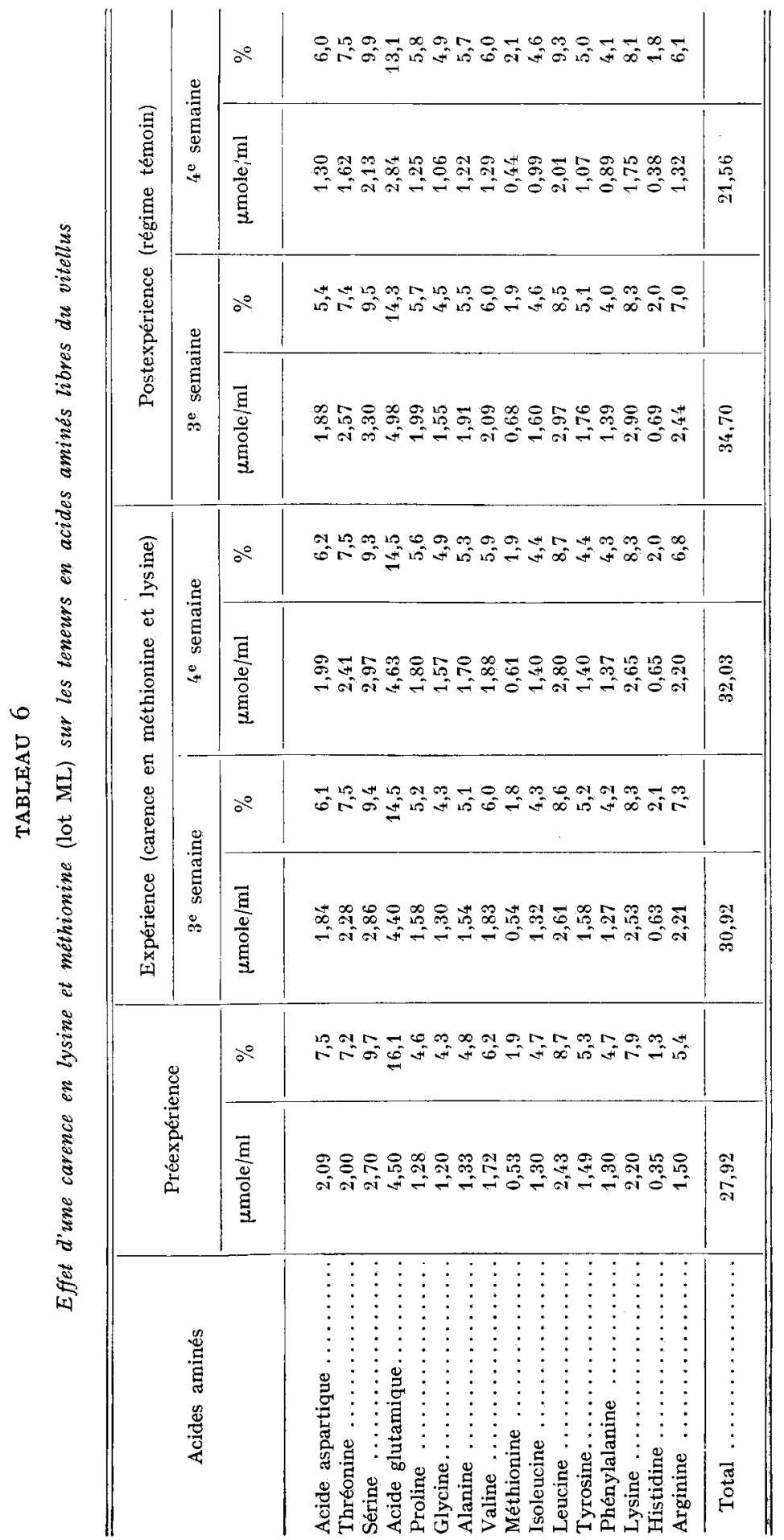


L'influence des carences en acides aminés chez la poule pondeuse a fait l'objet de nombreux travaux. Selon KrautmanN (I97I), un apport de protéines insuffisant a un effet dépendant de la souche : diminution du poids corporel, réduction du nombre ou du poids des œufs pondus, ces diverses manifestations étant ou non associées. Nos résultats montrent qu'il faut aussi tenir compte de la nature de l'acide aminé manquant. Mais il est nécessaire de nuancer les conclusions qui ressortent de la comparaison de l'effet des deux déficiences en méthionine et en lysine. La sévérité de la première pourrait expliquer à elle seule la réduction du développement corporel et de l'intensité de ponte. Les variations relatives du poids des constituants de 1'cuf fournissent une autre base d'appréciation. Proportionnellement, les œufs du lot $M$ contiennent moins d'albumen, les œufs du lot $L$ moins de vitellus. Ainsi, nous confirmons les observations de MORAN (I969) et de LECLERCQ (I970) : la déficience en méthionine réduit essentiellement l'albuminogenèse, la déficience en lysine amoindrit surtout la vitellogenèse. Ces effets pourraient être reliés aux teneurs en acides aminés des protéines de l'œuf : l'albumen, plus riche en méthionine, serait particulièrement affecté par la carence en cet acide aminé.

L'association de deux carences peut d'ailleurs modifier les résultats obtenus. Dans le lot ML où la méthionine est l'acide aminé le plus déficient, les diminutions du poids de l'œuf et du développement corporel sont paradoxalement atténuées par la carence partielle en lysine. En conservant son appétit, l'animal réussit à atténuer, par le jeu d'une meilleure utilisation de l'aliment ou d'une mobilisation de ses protéines tissulaires, les effets de la déficience en méthionine. Les deux déficiences intervenant à un niveau différent, albuminogenèse ou vitellogenèse, leur association rétablirait une sorte d'équilibre favorable à l'ovogenèse (JAAP, I969). Cependant, il faut noter le caractère provisoire de cette amélioration. Pendant la réhabilitation, les réserves tissulaires seraient reconstituées tandis que 1'albuminogenèse reste particulièrement déprimée témoignant d'un déficit en méthionine dont l'effet, pour être moins accentué pendant la période de carence, s'est prolongé dans le temps.

La diminution des performances de ponte pourrait être une conséquence secondaire des insuffisances d'apport de méthionine ou de lysine. En effet, toute déficience protéique réduit l'appétit des animaux. De ce fait, les nutriments disponibles pour l'ovogenèse deviennent moins abondants. Malgré le caractère imprécis des mesures de consommation, on constate bien une relation entre les performances et le niveau d'ingestion. Mais, c'est là une banalité et il est impossible de reconnaître le facteur intervenant en premier pour réđuire la ponte : manque de l'acide aminé lui-même ou des autres nutriments.

La mise en évidence et le dosage des acides aminés libres du vitellus apportent des renseignements originaux méritant quelques commentaires. Les teneurs du vitellus paraissent être maximales lorsque le régime est équilibré. Les valeurs enregistrées sont voisines, quoique légèrement inférieures à celles rapportées antérieurement par DUCAY, KIINE et MANDELEss (I960) et par FITZSImmons et WAIBEL (I967). La faible différence peut tenir aussi bien au mode d'extraction des acides aminés qu'aux conditions d'élevage. A la suite de BRAY et KELI,Y (I966), nous constatons que la teneur en lysine libre diminue en même temps que l'apport alimentaire. Ia diminution est même plus marquée que ne le laissait prévoir leur équation. Ces auteurs ne se sont préoccupés que de la lysine à l'exclusion des autres acides aminés. 
Ce faisant, ils n'ont pas enregistré la diminution conjointe qui affecte à peu près également tous les acides aminés libres. Une autre constatation originale ressort de notre essai : la déficience en méthionine intervient, comme dans le cas de la lysine, en réduisant quantitativement tous les acides aminés libres ; 1'appauvrissement en méthionine étant légèrement plus marqué. En définitive, compte tenu de ces résultats, on peut supposer que la diminution de teneur d'un acide aminé déterminé peut résulter de n'importe quel déséquilibre protidique dans la ration. Ainsi le « pool " d'acides aminés libres du vitellus ne semble pas caractériser de manière spécifique la nature du déficit en acides aminés dans 1'aliment, comme le font ceux du plasma et du muscle. Pour ces derniers, l'importance des modalités alimentaires a été récemment mise en évidence chez le Rat (STOCKLAND, MEAdE et MELLIERE, I970), et le Poulet (LARBier, Guillaume et Blum, I97I). Sans aucun doute, le niveau d'ingestion a pu jouer un rôle dans les variations de la teneur du vitellus en acides aminés libres. Cependant, on peut souligner l'absence d'une relation simple entre ces variations et la quantité d'aliment consommée.

L'appauvrissement en acides aminés libres du vitellus peut refléter l'état d'autres compartiments, en particulier ceux où s'effectuent des synthèses protidiques en relation avec l'ovogenèse. L'activité de synthèse pourrait être maintenue dans la mesure où le déficit est comblé par l'apport tissulaire. L'organisme de la Poule sacrifierait ses réserves lors d'une déficience en méthionine, et à un moindre degré, lorsque sont associées les carences en lysine et méthionine. Dans ce dernier cas la diminution de l'anabolisme de ponte est à la fois atténuée et prolongée dans le temps ; les teneurs en acides aminés libres diminuent tardivement lorsque se reconstituent les réserves tissulaires.

\section{CONCLUSION}

Les protéines tissulaires pourraient subvenir temporairement aux besoins de la Poule pondeuse manquant d'un ou plusieurs acides aminés indispensables. La mobilisation des réserves dépend non seulement de la souche (KRAUTMANN, I97I) mais aussi de 1'équilibre de la ration et de l'acide aminé manquant. Lorsque le défaut de 1'apport alimentaire est corrigé, les performances de ponte peuvent rester déprimées tant que les réserves tissulaires ne sont pas totalement reconstituées. Ainsi, un apport insuffisant d'acides aminés retentit sur l'ovogenèse après un délai variable. Il peut encore manifester son effet lorsque le régime est redevenu complet.

La teneur du vitellus en acides aminés libres paraît surtout refléter le métabolisme des protéines tissulaires. La diminution de cette teneur résulterait aussi bien d'un renouvellement ralenti par une carence en acide aminé indispensable, que d'un anabolisme accru pendant la reconstitution des réserves. Quoi qu'il en soit, une déficience modérée de lysine ou de méthionine appauvrit le vitellus en acides aminés libres sans altérer sensiblement leurs proportions relatives. La nature des protéines alimentaires influence donc la composition de l'œuf en constituants qui sont certes mineurs sur le plan quantitatif, mais dont le rôle peut être important pendant 1'incubation (KLEIN, MC CoNNEL, et Buckingham, I962). En tous cas, si dans nos 
conditions expérimentales, le dosage des acides aminés libres du vitellus ne révèle pas la nature exacte de la déficience, il constitue cependant un moyen pour déceler une insuffisance d'apport en acides aminés essentiels.

Reçu pour publication en août 1971.

\title{
SUMMARY
}

\section{EFFECT OF DEFICIENCY OF LYSINE AND METHIONINE}

IN THE FEED ON LAYING PERFORMANCE AND CONTENT

\author{
OF FREE AMINO ACIDS IN EGG YOLK
}

Pullets 30 weeks old were reared in individual cages. For a month (pre-experimental period) they had the same complete, balanced control diet. It was based on maize and groundnut meal and had pure L-lysine and DL-methionine added.

For the experiment the pullets were in 4 groups of 8 . The control group continued on the control diet ; groups M, L and ML had the diet made deficient in methionine ( 0.22 per cent methionine), in lysine ( 0.46 per cent lysine) or in both, by omission of these amino acids. After 4 weeks there was a post-experimental period during which all the pullets were again given the same control diet.

At the end of the experimental period egg production was reduced by 4.4 and 6.4 and in groups M and ML, respectively but not in group L. In the same order of groups egg weight was 8.0, 5.3 and $2.9 \mathrm{~g}$ less than in the control group. Proportionately the deficiency of methionine reduced weight of albumen and deficiency of lysine reduced that of yolk. Laying performance during the experimental period was better in group $M L$ than in group $M$, but remained depressed for longer in the post-experimental period.

After 2 or 3 weeks the deficient diets had changed the contents of free amino acids in the yolk. Quantitative changes concerned all the free amino acids almost equally. In groups $M$ and $L$ there was a decrease of between 25 and 50 per cent and in group ML the contents increased I 4 per cent. After 3 weeks in the post-experimental period concentrations of amino acids in groups $M$ and $L$ returned to normal values the same as in controls while in group ML they diminished by 25 per cent.

The balance of amino acids in the diet could influence the renewing of tissue protein and from that, the anabolism of laying and the concentration of free amino acids in egg yolk.

\section{RÉFÉRENCES BIBLIOGRAPHIQUES}

Bцum J. C., г966. Contribution à l'étude du métabolisme de la Riboflavine au cours de l'ovogenèse de Gallus gallus L. Thèse Doct. Sci., 162 p., Paris.

Bray D. J., KELLY M., I966. The effect of dietary lysine upon the free lysine content of egg yolks. Poult. Sci., 45, 1425-I426.

Ducay E. D., Kline L., Mandeless S., 1960. Free amino acid content of infertile chicken eggs. Poult. Sci., 39, 831-835.

Emanuelsson H., 1951. Proteolytic activity in hen's egg prior to incubations. Nature, 168, 958 -959.

Fitzsimmons R. C., Waibel P. E., ig68. Free amino acid composition of the avian egg as related to embryonic development. Poult. Sci., 47, 219-224.

Hamilton P. B., I962. Ion exchange chromatography of amino acids. Microdetermination of free amino acids in serum. Ann. N. Y. Acad. Sci., 102, 55-75.

HoRn M. J., Jones D. B., I945. A rapid colorimetric method for the determination of tryptophane; in proteins and foods, J. Biol. Chem., 157, I53-I60.

JAAP R. G., I969. Large broilers from smaller hens. World's Poult. Sci. J., 25, r40-143.

JONES J. D., I964. Lysine-Arginine antagonism in the chick. J. Nutr., 84, 3r3-32r.

Klein N. W., Mc Connel E., Buckingham B. J., 1962. Growth of explanted chick embryos on a chemically defined medium and effects of specific amino acid deficiencies. Develop. Biol., 5, 296-308. 
Krautmann B. A., I971. Genetics-nutrient interactions in laying hens. Fedn. Proc., 80, II8-120.

Larbier M., Guillaume J., Blum J. C., I97I. Muscle levels of free lysine and methionine in chicks fed a single daily meal. Nutr. Rep. Int., 3, 273-276.

Lea C. H., Rhodes D. N., I953. Phospholipids I. Partition chromatography of egg yolk phospholipids on cellulose. Biochem. J., 54, 467-469.

LeclercQ B., 1970. Facteurs nutritionnels modifiant le poids de l'œuf et de ses constituants. Ann. Biol. anim. Bioch Biophys., 10, 239-252.

Moran E. T., I969. Levels of dietary protein needed to support egg weight and laying hen production. Feedstuffs, 41, 22-30.

National Research Couvcil, I966. Nutrient requirements of poultry. Publication, 1345.

Pawlak M., Pion R., I968. Influence de la supplémentation des protéines de blé par des doses croissantes de lysine sur la teneur en acides aminés libres du sang et du muscle du Rat en croissance. Ann. Biol. anim. Bioch. Biophys., 8, 517-530.

Piez K. A., Morris L., I960. A modified procedure for the automatic analysis of amino acids. Ann. Biochim., 1, I87.

Pucher G. W., 1927. Chemical analysis of incubated non fertile eggs. Proc. Soc. Exptl. Biol. Med. 25, 72-73.

Richardson L. R., Blaylock L. G., Lyman C. M., I953. Influence of dietary amino acid supplements on the free amino acids in the blood plasma of chicks. $J$. Nutr., 51, 515-522.

Romanoff A. L., Romanoff A. J., 1949. The Avian Egg. John Wiley and Sons, Inc., New York.

Stockland W. L., Meade R. J., Melliere A. L., x97o. Lysine requirement of the growing Rat : Plasma free lysine as a response Criterion. J. Nutr., 100, 925-934.

Tallan H. H., Moore S., Stein W. H., r954. Studies on the free amino-acids and related compounds in the Cat. J. Biol. Chem., 211, 927-939.

Williams M. A., Da Costa W. A., Newman L, H., Marshall L. M., 1954. Free amino acids in the yolk during the the development of the chick. Nature, 173, 49 . 